\title{
Indicadores de desempenho em instituições de ciência, tecnologia e inovação: estudo de caso do Laboratório Nacional de Luz Síncrotron
}

\author{
Eduardo Frare \\ Pontifícia Universidade Católica de Campinas \\ Ricardo Lopes Cardoso \\ Universidade Presbiteriana Mackenzie \\ José Carlos Tiomatsu Oyadomari \\ Universidade Presbiteriana Mackenzie e Insper Instituto de Ensino e Pesquisa \\ Octavio Ribeiro de Mendonça Neto \\ Universidade Presbiteriana Mackenzie
}

\begin{abstract}
Este estudo trata de indicadores de desempenho em organizações de Ciência, Tecnologia e Inovação (C, T \& I) e, em particular, do Laboratório Nacional de Luz Síncrotron (LNLS). Teve por objetivo determinar os indicadores a serem utilizados pelo LNLS para garantir o atingimento de seus objetivos de curto e longo prazo. Para tanto foi realizado um estudo de caso. Os dados foram coletados por meio de entrevistas semiestruturadas junto a 10 stakeholders com elevado grau de influência e interesse na instituição, analisados pela técnica de análise de conteúdo e triangulados por meio da análise de documentos e da observação participante. Como resultado, este estudo sugere um modelo de acompanhamento de desempenho a ser adotado pelo LNLS que também pode ser útil para as demais organizações de C, T \& I.
\end{abstract}

Palavras-chave: indicadores de desempenho; Laboratório Nacional de Luz Síncrotron; ciência, tecnologia \& inovação.

Indicadores de desempeño en instituciones de ciencia, tecnología e innovación: un estudio de caso del Laboratorio Nacional de Luz Sincrotrón

Este estudio trata de los indicadores de desempeño en organizaciones de Ciencia, Tecnología e Innovación (C, Te \& I) y, en particular, del Laboratorio Nacional de Luz Síncrotron (LNLS). Su objetivo

DOI: http://dx.doi.org/10.1590/0034-76121633

Artigo recebido em 4 jul. 2013 e aceito em 8 maio 2014.

Rev. Adm. Pública - Rio de Janeiro 48(5):1229-1252, set./out. 2014 
fue determinar los indicadores que se utilizarán por el LNLS para asegurar el logro de sus metas a corto y largo plazos. Por lo tanto, se realizó un estudio de caso. Los datos fueron recopilados a través de entrevistas semi-estructuradas con 10 participantes con alta influencia e interés por la institución, analizados por la Técnica de Análisis de Contenido y triangulados a través del análisis de documentos y observación participante. Como resultado, este estudio sugiere un modelo de supervisión del rendimiento para ser adoptado por LNLS, que también puede ser útil para otras organizaciones de C, T \& I.

Palabras clave: indicadores de desempeño; Laboratorio Nacional de Luz Sincrotrón, ciencia, tecnología \& innovación.

Performance indicators in institutions of science, technology and innovation: a case study of the National Synchrotron Light Laboratory

This study deals with performance indicators in organizations of Science, Technology and Innovation (S, T \& I) and, in particular, the National Synchrotron Light Laboratory (NSLL). It aimed to determine indicators to be used by NSLL to ensure the achievement of its objectives of short and long term. To do so, we performed a case study. The data were collected through semi-structured interviews with 10 stakeholders with high influence and interest in the institution, analyzed by the Analysis of Content's technique and triangulated through document analysis and participant observation. As a result, this study suggests a model of performance management to be adopted by NSLL that can also be useful for other organizations of S, T \& I.

KEYWORDs: performance indicators; National Synchrotron Light Laboratory; science, technology \& innovation.

\section{Introdução}

Este estudo trata de indicadores de desempenho em organizações de ciência, tecnologia e inovação (C, T \& I), as quais são relevantes para impulsionar o desenvolvimento econômico. Entende-se como desenvolvimento econômico "(...) o crescimento sustentável da renda per capita e do emprego, associado à melhoria da distribuição da renda de pessoal e regional e à conservação do meio ambiente". (Brasil, 2001:119).

$\mathrm{Na}$ medida em que a obtenção e a aplicação do conhecimento resultante da ciência, da tecnologia e do processo de inovação demandam investimentos elevados, o acompanhamento dos resultados obtidos se impõe, mormente em países em desenvolvimento como o Brasil, onde a maior parte desses investimentos é financiada com recursos públicos.

A preocupação com o acompanhamento dos resultados das organizações de C, T \& I tem levado ao desenvolvimento de novos modelos de gestão, os quais têm sido propostos com o objetivo de gerenciar as atividades dessas organizações de forma mais eficiente e ampliar sua legitimidade junto à sociedade.

Apesar de enfrentarem desafios comuns, essas instituições têm adotado estruturas distintas, dentre elas se destaca o modelo denominado de organização social (OS), criado no contexto do programa de Reforma do Estado no final da década de 1990, do qual o Laboratório Nacional de Luz Síncrotron (LNLS), objeto do presente estudo de caso, foi pioneiro na sua adoção. 
A adoção do modelo de OS impactou na forma como o desempenho dessas entidades passou a ser medido e avaliado e visou também, por meio de um sistema específico de mensuração do desempenho institucional, ampliar a legitimidade das mesmas diante dos seus stakeholders. Do ponto de vista da legitimidade, nota-se uma evolução nas formas de produção de conhecimento científico e tecnológico e de geração de inovações, tais como o controle da qualidade da produção científica e tecnológica. Por outro lado, também é necessário apresentar justificativas para investimentos em pesquisa e desenvolvimento (P\&D) já que existem demandas sociais por controle, coordenação e desenvolvimento de mecanismos de governança para estas atividades (Bin, 2008).

A mensuração de desempenho nesse tipo de instituição foge dos modelos tradicionais baseados em valor de mercado e em informações contábeis (Merchant e Van Der Stede, 2007) tornando-se mais complexa, uma vez que, em organizações de C, T \& I, predominantemente sem finalidade lucrativa, o desempenho pode ser captado de outras perspectivas, com ênfase no valor intangível de suas atividades, e tem seu desempenho monitorado por diferentes stakeholders, conforme Ferreira e Otley (2009) e Malmi e Brown (2008), por estar inserido em um contexto de organização social.

Partindo do pressuposto teórico de que o papel do sistema de controle gerencial é influenciar o comportamento dos gestores em prol dos objetivos organizacionais (Malmi e Brown, 2008), surge um dilema sobre o horizonte temporal de desempenho, especificamente quanto aos objetivos de longo prazo versus objetivos de curto prazo. Esse dilema existe porque, embora o desempenho financeiro não seja o objetivo principal dessas organizações, ele é necessário, uma vez que as mesmas não conseguiriam sobreviver caso as receitas fossem menores do que as despesas, já que não têm autonomia gerencial para contratar empréstimos.

Tipicamente, os resultados científicos e tecnológicos das organizações de C, T \& I são obtidos no longo prazo. Portanto, a sistemática de acompanhamento do desempenho dessas organizações deve considerar também um conjunto de indicadores que possa sinalizar a capacidade de geração desses resultados no futuro.

Nesse contexto, o problema central desta pesquisa pode ser sintetizado na seguinte questão: quais indicadores de desempenho devem ser utilizados pela direção do Laboratório Nacional de Luz Síncrotron para assegurar o cumprimento de sua missão e garantir o atingimento de objetivos de curto e longo prazo?

Este estudo prossegue com a apresentação do referencial teórico e na sequência são descritos os procedimentos de coleta de dados e análise dos mesmos. Por fim, são apresentadas as considerações finais, baseadas em um modelo conceitual proposto pelos autores.

\section{Referencial teórico}

O referencial teórico do presente trabalho abrange o ambiente de ciência, tecnologia e inovação, sistema de controle gerencial, indicadores de desempenho e incentivos. 


\subsection{Ambiente de ciência, tecnologia e inovação}

No ambiente de C, T \& I, dois conceitos centrais marcam o entendimento atual a respeito da natureza e organização da atividade científica e tecnológica: o de não linearidade e o de inovação. Schwartzman (2002) afirma que a noção de que as atividades de pesquisa e desenvolvimento obedecem a uma sequência linear - da pesquisa básica à pesquisa aplicada, desta ao desenvolvimento tecnológico, e deste, finalmente, ao produto de uso prático — não é a que melhor descreve o que ocorre no mundo real. Observa que a literatura especializada sugere a existência de uma "sequência invertida", que tem início com atividades de inovação de produtos culminando no desenvolvimento de pesquisa experimental e básica. Ressalta ainda que as atividades de pesquisa básica, experimental e o desenvolvimento de produtos se dão de forma simultânea e imprevisível, que incluem todas as etapas relevantes da cadeia de geração-produção de conhecimentos e produtos (Schwartzman, 2002:374). Diante desse contexto caracterizado pela complexidade e pelo alto grau de incerteza, a teoria da contingência sugere que essas organizações deveriam adequar sua estrutura organizacional a seus fatores contingenciais e assim ao ambiente, de modo a obter um nível adequado de desempenho organizacional (Donaldson, 2007).

Há ainda que se considerar que nas atividades de C\&T existe a necessidade de repensar os procedimentos e mecanismos utilizados pela sociedade para avaliar e justificar os investimentos realizados (Schwartzman, 2002), tendo em vista as diferentes propostas de produção do conhecimento, conforme destacam Gibbons e colaboradores (1994). O desempenho de produção do conhecimento pode ser acompanhado por meio de publicações científicas, mas continuam sendo importantes para a ciência aplicada o uso e a satisfação do usuário como indicadores dominantes de desempenho.

Dessa forma, embora medidas como o lucro estejam ausentes, a captação de recursos de órgãos de fomento, sejam privados ou públicos, pode ser um reflexo da avaliação de desempenho na perspectiva dos stakeholders externos.

\subsection{Sistemas de controle gerencial (SCG)}

Os sistemas de controle gerencial (SCG) têm sido definidos como o conjunto de práticas, procedimentos, sistemas e ferramentas que a organização utiliza para influenciar o comportamento dos gerentes a atingirem os objetivos organizacionais (Merchant e Van Der Stede, 2007; Malmi e Brown, 2008). Em organizações privadas, os objetivos organizacionais mais importantes são geralmente mensurados em termos monetários, mas em organizações sem fins lucrativos a ausência de lucro é a regra (Anthony e Govindarajan, 2006).

Nesse sentido, até o processo de controle deveria ser ajustado à luz das características dessas organizações, pois, conforme preconiza a teoria da contingência, em organizações onde predominam tarefas de baixa incerteza, estas são executadas mais eficazmente por meio de uma hierarquia centralizada, enquanto naquelas onde a incerteza das tarefas aumenta, a eficácia decorre da inovação. Desta forma, a hierarquia precisa reduzir o controle e incentivar estruturas comunicativas e participativas (Donaldson, 2007), uma vez que o conhecimento 
necessário ao desempenho das tarefas nestas organizações está diluído entre os seus empregados (Burns e Stalker, 1961).

A literatura mais aderente a organizações de C\&T é a abordagem apresentada por Chiesa e colaboradores (2007) para empresas com áreas/departamentos dedicados a atividade de pesquisa e desenvolvimento. Nessa abordagem, os autores reafirmam a necessidade de adequação dos sistemas de controle gerencial na perspectiva organizacional, enquanto uma área da organização. No presente estudo entende-se que essa abordagem pode também ser utilizada para organizações de C, T \& I na medida em que o ambiente de pesquisa e de inovação dessas também é caracterizado por elevado nível de incerteza.

Alguns dilemas presentes em organizações privadas relacionados com o sistema de controle gerencial são operacionalizados pelo conceito de tensões dinâmicas (Simons, 2005), as quais, de acordo com Oyadomari (2008:32), podem ser definidas como dilemas organizacionais que apresentam visões conflitantes sobre os objetivos organizacionais. A seguir, são discutidas aquelas pertinentes às organizações de C, T \& I.

- O adequado balanceamento entre competição e cooperação (Etherington e Tjosvold, 1998), em organizações de C, T \& I, deve gerar benefícios ao processo de aprendizado. No entanto, sua aplicação em equipes de alto desempenho pode se tornar complexa na medida em que o estímulo a desempenhos melhores pode resultar em um efeito negativo na cooperação.

- O crescimento das organizações versus o risco também pode ser um dilema presente, pois o crescimento é importante, e bastante valorizado, em função do aumento de seu poder de barganha junto a fornecedores e clientes (Porter, 1989), e, também, da redução de custos devido a economias de escala, ou ainda da legitimidade junto aos stakeholders. Todavia, mesmo o crescimento orgânico pode representar uma forte ameaça devido à ampliação dos riscos organizacionais, uma vez que há também a frequente limitação de recursos humanos qualificados, o que leva a um acúmulo excessivo de atividades para a equipe de pesquisadores da organização.

- O dilema entre resultados de curto e longo prazo é o mais evidente, pois apesar de as atividades de pesquisa e desenvolvimento focarem, de uma forma geral, nos resultados de médio e longo prazo, as etapas de concepção e implementação de novos experimentos devem ser minuciosamente planejadas e impactam as ações de curto prazo.

- Por fim, a tensão dinâmica entre foco interno versus foco externo também está presente nessas organizações devido à busca constante por legitimidade social, o que pode vir a se confrontar com o interesse específico da pesquisa propriamente dita.

\subsection{Indicadores de desempenho e incentivos}

Diversos frameworks de sistema de controle gerencial como os propostos por Malmi e Brown (2008), Ferreira e Otley (2009), Kaplan e Norton (1996) e Neely e colaboradores (2002) colocam como ponto importante o tema "indicadores de desempenho".

Indicadores de desempenho são úteis para avaliar o cumprimento dos objetivos estratégicos, o andamento dos fatores críticos de sucesso e o desempenho, com a consequente influência no comportamento das pessoas (Ferreira e Otley, 2009). 
Esses indicadores também têm objetivos informacionais e são utilizados para avaliar o desempenho em diferentes níveis organizacionais (Franco-Santos, Lucianetti e Bourne, 2012).

Quando as organizações usam uma combinação de indicadores de desempenho monetários e não monetários, conforme preconizam Kaplan e Norton (1996), pode-se dizer que, à luz da literatura, essas organizações estão utilizando os sistemas híbridos (Malmi e Brown, 2008) ou ainda sistemas contemporâneos de mensuração de desempenho (Franco-Santos, Lucianetti e Bourne, 2012).

A respeito dos sistemas contemporâneos de desempenho, Franco-Santos, Lucianetti e Bourne (2012), em uma extensa revisão da literatura, classificaram os estudos relacionados com o tema em quatro perspectivas: (1) efeitos sobre o comportamento; (2) consequências para as competências organizacionais; (3) consequências para o desempenho; e, por fim, estudaram as (4) teorias relacionadas com esses estudos.

Para o presente referencial teórico, foram explorados alguns aspectos relacionados com as três primeiras perspectivas consoante o objetivo de pesquisa. Sobre os efeitos no comportamento das pessoas, a literatura destaca que os sistemas de medição de desempenho melhoram as discussões no nível dos executivos, habilitando-os a concentrarem seus esforços naquilo que é realmente importante. Outro aspecto é o efeito na cooperação, coordenação e participação, e também na motivação. Já sobre as competências, os estudos têm apontado as diversas formas como o uso interativo produz efeitos na inovação e na aprendizagem organizacional, enquanto sobre o efeito no desempenho os estudos ainda não são conclusivos. Estudos com abordagem quantitativa encontraram relacionamentos positivos com o desempenho (medido pela percepção dos gestores), o que não tem sido confirmado em estudos qualitativos (FrancoSantos, Lucianetti e Bourne, 2012).

Todavia, tanto a literatura sobre os sistemas de controle gerencial como a literatura sobre os sistemas contemporâneos de desempenho têm enfatizado a questão dos incentivos, seja como parte do sistema de controle gerencial (Malmi e Brown, 2008), seja como fatores de motivação extrínseca (Ferreira e Otley, 2009), ou ainda relacionando os incentivos com a percepção dos colaboradores sobre a questão da justiça organizacional (Franco-Santos, Lucianetti e Bourne, 2012).

Porém, em organizações de C, T \& I, os incentivos monetários diretos praticamente inexistem, portanto, essas organizações dependem mais de fatores intrínsecos e os incentivos são basicamente o reconhecimento por pares de que os desempenhos da organização ou do grupo de pesquisa são superiores e comparáveis com os níveis de fronteira. Em particular para o LNLS, esse reconhecimento se reflete pela renovação do contrato de gestão com o Ministério de Ciência e Tecnologia e pela ampliação de sua importância no Sistema Nacional de Ciência, Tecnologia e Inovação.

A literatura acadêmica baseada em livros texto é farta em mostrar diversos casos de proposta de indicadores de desempenho, mas trabalhos científicos são escassos. Uma exceção é o trabalho de Malina e Selto (2004), os quais desenvolveram um estudo em uma indústria norteamericana e propuseram um sistema de indicadores de desempenho para a cadeia de distribuidores dessa organização. O modelo de mensuração de desempenho por eles proposto pretendeu 
refletir o entendimento da organização e das relações de desempenho entre os recursos estratégicos, capacidades operacionais e resultados desejados, valorizando a questão da escolha das medidas de desempenho e destacando ainda que esses indicadores devem ser alterados para refletir as condições atuais do ambiente em que a organização está inserida (Malina e Selto, 2004). Essa concepção é aderente para organizações de C, T \& I devido a suas características peculiares e, portanto, alinhada com a questão de pesquisa levantada neste estudo.

\section{Procedimentos metodológicos}

\subsection{Características do LNLS}

O LNLS inaugurou suas instalações experimentais em julho de 1997 e, atualmente, caracteriza-se como um centro nacional de pesquisas multidisciplinares, aberto a profissionais qualificados de qualquer nacionalidade, de várias áreas do conhecimento.

Atualmente, o Contrato de Gestão firmado entre o LNLS e o Ministério de Ciência e Tecnologia (MCT) é o principal instrumento de regulação dos deveres e obrigações da entidade. Nesse contrato estão descritos os objetivos estratégicos da organização, as expectativas do órgão supervisor com relação ao papel da entidade no sistema nacional de C\&T e o painel de indicadores de desempenho com metas anuais, mas com acompanhamento semestral pela Comissão de Avaliação designada pelo órgão supervisor.

O órgão não adota nenhum sistema de incentivos para os gestores ou colaboradores uma vez que a administração entende que essa não é uma prática habitual desse segmento e também por questões de natureza regulatória e legal, e o único incentivo para o cumprimento do desempenho é a renovação do Contrato de Gestão.

Os três macro-objetivos previstos nesse contrato são: a) atuar como laboratório nacional, b) realizar pesquisa própria, desenvolvimento e inovação; e c) implementar novos mecanismos de gestão, informação e difusão de C, T \& I.

Na tabela 1 é apresentada uma síntese do orçamento previsto na Lei Orçamentária Anual (LOA) para as atividades de operação do LNLS, e dois dos indicadores pactuados para o período de 2011-16.

Tabela 1

Orçamento 2011-16

\begin{tabular}{|lrrrrrr|}
\hline \multicolumn{7}{|c|}{ Contrato de gestão - orçamento e metas pactuadas } \\
\hline \multicolumn{1}{|c}{ Ano } & 2011 & 2012 & 2013 & 2014 & 2015 & 2016 \\
\hline Orçamento - LNLS & & & & & & \\
(em R\$ mil) & 34.350 & 36.068 & 37.871 & 39.765 & 41.753 & 43.841 \\
N. propostas & 870 & 955 & 970 & 1.025 & 1.025 & 1.025 \\
N. publicações & 280 & 310 & 330 & 357 & 357 & 357 \\
\hline
\end{tabular}

Fonte: Contrato de gestão 2011-16. 
A seguir são descritos os diferentes fóruns da Estrutura Organizacional:

Conselho de Administração: é formado por membros indicados pelo poder público e por representantes da sociedade civil organizada. Reúne-se pelo menos a cada quatro meses para analisar e aprovar os relatórios de prestação de contas da entidade.

Comitê Científico: Scientific Committee se reúne a cada dois anos e divulga um relatório final com críticas e sugestões.

Diretoria Executiva: responsável pela execução das políticas estratégicas definidas pelo Conselho de Administração.

Líderes e Funcionários: funcionários contratados pela organização em sua maioria em tempo integral. Em geral, possuem formação qualificada e especializada nas áreas de engenharia, física, química ou biologia, além do quadro técnico e administrativo.

Ministério da Ciência e Tecnologia e Comissão de Acompanhamento e Avaliação do Contrato de Gestão: principal provedor de recursos ao LNLS. As relações entre as partes estão convencionadas em um Contrato de Gestão, repactuado anualmente. A Comissão de Acompanhamento e Avaliação do Contrato de Gestão é quem monitora o desempenho pactuado no Contrato com o MCT.

\subsection{Técnicas de coleta de dados}

Os dados do presente estudo foram coletados por meio de entrevistas semiestruturadas, posteriormente analisados pela técnica de análise de conteúdo e triangulados por meio da análise de documentos e observação participante. A seleção dos entrevistados baseou-se no conjunto de stakeholders com elevado grau de influência e interesse na organização, representando a maior parte dos potenciais entrevistados.

\subsubsection{Elaboração do roteiro de entrevistas}

O roteiro de entrevista foi elaborado com base na literatura e incorporou sugestões de dois potenciais entrevistados e de um terceiro entrevistado. Os respondentes foram submetidos a 10 questões abertas e um quadro com os atuais indicadores de desempenho foi apresentado a eles.

\subsection{Dados dos entrevistados}

A tabela 2 descreve a distribuição dos entrevistados por categorias de responsabilidades. 
Tabela 2

\section{Composição da amostra}

\begin{tabular}{|c|c|c|}
\hline Categoria organizacional & $\begin{array}{c}\text { Quantidade de } \\
\text { entrevistados }\end{array}$ & Papel organizacional \\
\hline Dirigentes e ex-dirigentes & 4 & $\begin{array}{l}\text { Diretores e ex-diretores do LNLS responsáveis pela formulação } \\
\text { de estratégia organizacional e negociação das medidas de } \\
\text { desempenho com o Conselho de Administração e Ministério da } \\
\text { Ciência e Tecnologia. }\end{array}$ \\
\hline Líderes (técnico e de pesquisa) & 2 & $\begin{array}{l}\text { Gestores da organização, denominados líderes de grupo, } \\
\text { responsáveis pela implementação da estratégia organizacional e } \\
\text { execução de atividades de pesquisa e desenvolvimento. }\end{array}$ \\
\hline Órgão de supervisão & 1 & $\begin{array}{l}\text { O Conselho de Administração da organização responsável pela } \\
\text { aprovação da estratégia organizacional e monitoramento do } \\
\text { desempenho. }\end{array}$ \\
\hline Agentes de avaliação & 3 & $\begin{array}{l}\text { Membros do Comitê Científico, da Comissão de } \\
\text { Acompanhamento e Avaliação do Contrato de Gestão e ex- } \\
\text { ombdusperson, responsáveis por avaliações ou percepções } \\
\text { periódicas do desempenho da organização. }\end{array}$ \\
\hline
\end{tabular}

Fonte: Elaboração dos autores.

Foram entrevistados dois dirigentes atuais e dois ex-dirigentes, dois líderes do LNLS, o presidente do Conselho de Administração, um representante do Ministério supervisor, um membro do Comitê Científico e um ex-ombudsperson da entidade.

Na tabela 3 são identificados os entrevistados, a data e a duração das entrevistas.

Tabela 3

Relação dos entrevistados

\begin{tabular}{|clcc|}
\hline & \multicolumn{1}{c}{ Função } & $\begin{array}{c}\text { Data da } \\
\text { entrevista }\end{array}$ & $\begin{array}{c}\text { Duração } \\
\text { em horas }\end{array}$ \\
\hline LG1 & Líderes e pesquisador & $10 / 03 / 2010$ & $00: 55: 04$ \\
LG2 & Líderes e pesquisador & $29 / 01 / 2010$ & $01: 22: 17$ \\
DL3 & Dirigentes e ex-dirigentes & $22 / 03 / 2010$ & $00: 53: 53$ \\
DL4 & Dirigentes e ex-dirigentes & $24 / 03 / 2010$ & $00: 52: 22$ \\
CA5 & Órgão de supervisão & $20 / 04 / 2010$ & $00: 40: 00$ \\
AV6 & Agentes de avaliação & $23 / 04 / 2010$ & $00: 45: 00$ \\
US7 & Agentes de avaliação & $30 / 03 / 2010$ & $00: 58: 48$ \\
CC8 & Agentes de avaliação & $07 / 04 / 2010$ & $00: 49: 46$ \\
DL9 & Dirigentes e ex-dirigentes & $09 / 03 / 2010$ & $01: 14: 15$ \\
DL10 & Dirigentes e ex-dirigentes & $08 / 03 / 2010$ & $00: 30: 00$ \\
\hline
\end{tabular}

Fonte: Elaboração dos autores. 


\subsection{Procedimentos de tratamento de dados}

A análise dos dados contemplou a identificação dos pontos-chave, quando foi elaborada uma matriz de pontos-chave versus perguntas. Essa matriz foi elaborada a partir do painel de indicadores do LNLS que foram classificados, com base em Kaplan e Norton (1996), como parâmetros estratégicos - resultados e causais; financeiros e não financeiros; e natureza interna e externa à organização - e pelas perspectivas de avaliação - financeira; processos internos e operacionais; aprendizado e inovação; e clientes/usuários.

Após a "leitura flutuante", ou seja, quando os pesquisadores tomam um primeiro contato com os dados, e com base nas regras de exaustividade do material transcrito das entrevistas conforme a proposta de Bardin (2004), foram identificados como são medidos esses indicadores, com qual periodicidade e resumidas as dificuldades de sua mensuração.

O método de análise de conteúdo apresenta-se como adequado aos propósitos do presente trabalho devido ao objetivo primário de estudar as comunicações, com maior ênfase no conteúdo das mensagens, privilegiando a análise de dados qualitativos.

\subsection{Etapas da análise de conteúdo}

Bardin (2004) esclarece que a análise de conteúdo possui três etapas básicas: i) pré-análise, ii) exploração do material e iii) o tratamento dos resultados.

i) Pré-análise: no presente estudo, a pré-análise consistiu na organização do material, na escolha dos documentos a serem analisados, e na formulação de pressupostos que fundamentaram a interpretação final.

ii) Exploração do material: esta etapa consiste na codificação dos dados, ou seja, visa a descrever as características referentes ao conteúdo, com ênfase na escolha de categorias.

iii) Tratamento dos resultados: após as etapas de pré-análise e exploração do material, a análise de conteúdo foi aplicada para o tratamento de dados conforme esquema apresentado na figura 1.

\section{Apresentação e discussão dos resultados}

\subsection{Apresentação e discussão por categorias e unidades de significado}

Nos quadros 1 e 2 são apresentadas as unidades de significado, as categorias e os destaques dos trechos de entrevistas resultantes da análise de conteúdo. 
Figura 1

Esquema básico da análise de conteúdo

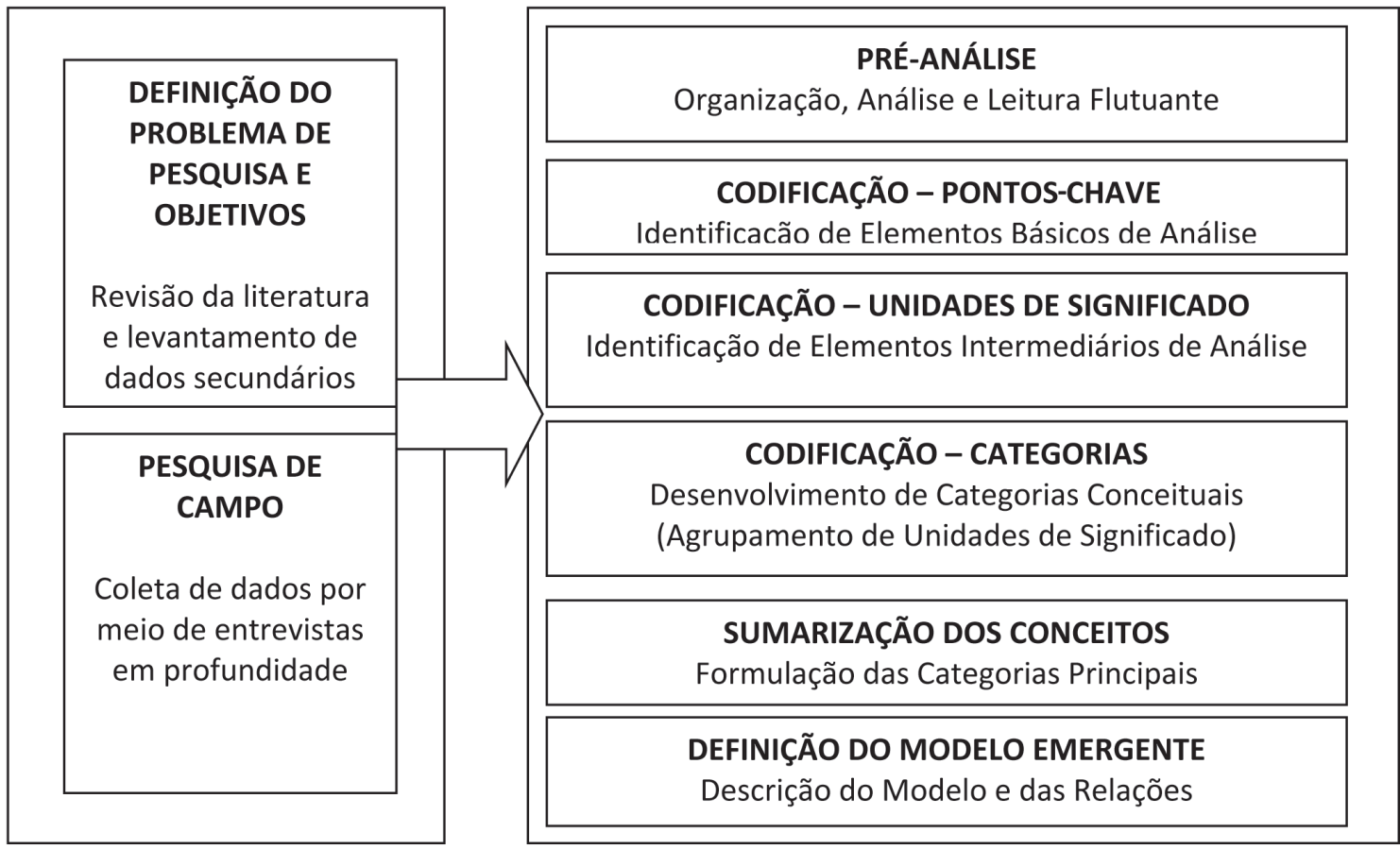

Fonte: Adaptado de Bardin (2004) e Neuendorf (2002), esquematizado por Perez (2006).

Quadro 1

Categorias criadas em função das unidades de significado identificadas nas entrevistas

\begin{tabular}{|ll|}
\hline \multicolumn{1}{|c|}{ Categorias } & \multicolumn{1}{c|}{ Unidades de significado } \\
\hline CAT 01 - Legitimidade institucional & 1. Foco da missão institucional \\
& 2. Monitoramento do ambiente de C\&T \\
& 3. Indução de novas áreas de atuação \\
& 4. Competitividade e cooperação internacional \\
CAT 02 - Resultados científicos & 6. Fronteira do conhecimento científico (ousadia) \\
& 7. Undependência e integração das avaliações externas \\
CAT 03 - Confiabilidade das instalações & 8. Pesquisa própria \\
CAT 04 - Disponibilidade das instalações & 9. Principal (Fonte de Luz Síncrotron) \\
& 10. Instalações correlatas \\
11. Laboratório Nacional & 12. Laboratório Aberto \\
CAT 05 - Recursos humanos & 13. Laboratório Multiusuário e multidisciplinar \\
& 14. Atração \\
& 15. Retenção \\
& 16. Capacitação
\end{tabular}




\begin{tabular}{|lll|}
\hline \multicolumn{1}{|c|}{ Categorias } & \multicolumn{1}{c|}{ Unidades de significado } \\
\hline CAT 06 - Recursos financeiros & 17. & Recursos do Contrato de Gestão \\
& 18. & Recursos gerados e captados \\
CAT 07 - Eficiência gerencial & 19. Modelo gerencial \\
& 20. Cenário de incertezas (capacidade de adaptação) \\
& 21. Atuação na geração do conhecimento \\
& 22. Difusão científica e tecnológica \\
\hline
\end{tabular}

Fonte: Elaborado pelos autores.

A Categoria CAT 01 — Legitimidade institucional relaciona-se fortemente com medidas de acompanhamento de estratégia da organização e de relevância nas suas áreas de atuação. Foi formada por seis unidades de significado, a saber:

a. Foco da missão institucional: foi identificado pela totalidade dos entrevistados como um fator-chave do sucesso do LNLS, principalmente pela clareza do papel que deve ser desempenhado pela instituição dentro do Sistema Nacional de Ciência, Tecnologia \& Inovação.

b. Monitoramento do Ambiente de C\&T: reflete a importância da interação dessas organizações com o ambiente externo visando ampliação de sua legitimidade com foco em sua missão institucional. A identificação de novas áreas de atuação, sejam científicas ou tecnológicas, sinaliza uma postura ativa da organização e deve refletir na competitividade da sua equipe e em maior interesse em cooperação por outras organizações.

c. Indução de novas áreas de atuação; parte da perspectiva de atender à demanda potencial da comunidade de pesquisa, desenvolvimento e inovação, seja pública ou privada, para resolver problemas estratégicos na área de atuação do LNLS.

d. Competitividade e cooperação internacional: ênfase na necessidade de a organização manter uma postura científica competitiva em relação a outros players, mantendo uma postura de forte cooperação e de muito diálogo com a comunidade, indústria e academia.

e. Fronteira do conhecimento científico (ousadia): importância declarada pelos entrevistados de se manter a ousadia científica, principalmente no desenvolvimento de pesquisa de fronteira. Quanto às medidas de acompanhamento, estas são consideradas complexas e indiretas, mas entendidas como necessárias para o monitoramento do desempenho da organização.

f. Independência e integração das avaliações externas; os entrevistados destacaram a importância das avalições científicas com comitês externos à instituição como elemento importante para ampliar a legitimidade institucional, sendo necessário maior integração e articulação entre esses agentes. Deve-se destacar ainda a importância de se comunicar adequadamente os resultados dessas avaliações e, principalmente, as ações adotadas pela organização para sanar as deficiências identificadas pelos respectivos avaliadores. 
A Categoria CAT 02 - Resultados científicos foi identificada pelas seguintes unidades de significado:

a. Resultados científicos produzidos por usuários externos: foi ressaltada pelos entrevistados a complexidade de medir os resultados científicos, e também a importância de avaliação qualitativa desses resultados produzidos por pesquisadores externos ao LNLS, sendo necessário o seu monitoramento ao longo do tempo.

b. Pesquisa própria: há a percepção da necessidade de se ter uma equipe interna atuante e com foco estratégico para gerar resultados qualitativos e elevar a capacidade da própria comunidade de usuários-pesquisadores externos.

A Categoria CAT 03 - Confiabilidade das instalações foi formada pelas seguintes unidades de significado:

a. Principal (Fonte de Luz Síncrotron): parâmetro de comparação internacional entre os Laboratórios Síncrotron do mundo; a confiabilidade da máquina é uma medida importante de credibilidade técnica em função da repercussão negativa que pode ser gerada quando as instalações não funcionam adequadamente e pela natureza do atendimento a usuários externos, inerente a laboratórios nacionais.

b. Instalações correlatas: a percepção de credibilidade da organização deve ser permanentemente monitorada. Em uma série de instalações de apoio para a realização dos experimentos com Luz Síncrotron, definidas como instalações correlatas, como a preparação de amostras, o laboratório de raios-X e de química, o monitoramento da confiabilidade não é sistemático, havendo, portanto, a necessidade de ampliação dessas medidas para além da Fonte de Luz Síncrotron.

A Categoria CAT 04 - Disponibilidade das instalações agrupou as seguintes unidades de significado:

a. Laboratório Nacional: a atuação como Laboratório Nacional é entendida como determinante para a legitimidade do LNLS. A ideia central é oferecer um conjunto de instalações complexas à comunidade científica, mantida por uma equipe técnica especializada e a serviço desses usuários-pesquisadores. Esse conceito tem permitido ampliar as alternativas de pesquisa científica na medida em que é possível se realizarem experimentos simultâneos, por usuários distintos e de forma multidisciplinar.

b. Laboratório Aberto: também foi ressaltado que apesar de ser nacional ele deve manter sua característica de Laboratório Aberto para toda a comunidade científica e tecnológica, e o acesso às instalações deve ser meritocrático.

c. Laboratório Multiusuário e Multidisciplinar: outros entrevistados apontam para a questão do laboratório ser multiusuário e multidisciplinar, ou seja, deve atender a um conjunto amplo de interessados e de forma multidisciplinar. 
A categoria CAT 05 - Recursos humanos apresentou três unidades de significado

a. Atração: era esperada a caracterização da categoria de recursos humanos, considerando a especificidade e especialidade da organização. A busca de estímulos para a atração de jovens pesquisadores foi apresentada como importante para contribuir com a ampliação da legitimidade do LNLS.

b. Retenção: é um aspecto importante em função do nível de especialização do corpo de pesquisadores, e com a ampliação do campo de atuação da organização, a perda de talentos é percebida como um risco de perder competências técnicas e científicas que não são facilmente repostas.

c. Capacitação: outro aspecto importante a ser observado é a necessidade de uma constante atuação para a capacitação de usuários em novas técnicas de pesquisa, a formação de novos usuários e a ampliação da competência da instrumentação científica.

A necessidade de se combinar diversas ações institucionais para atração, retenção e capacitação dos recursos humanos ficou evidente nas entrevistas. No entanto, não foram identificados indicadores formais para monitorar essas questões no LNLS.

A Categoria CAT 06 - Recursos financeiros foi agrupada nas seguintes unidades de significado e reflete a estrutura de financiamento do LNLS:

a. Recursos do Contrato de Gestão: esses recursos são mais previsíveis e visam manter a operação regular da organização, porém nota-se nas entrevistas uma grande insatisfação com o montante de recursos destinado à operação regular. Outros preferiram apontar para a questão da estabilidade orçamentária como base para uma operação adequada, inclusive com implicações para atratividade e manutenção de pesquisadores.

b. Recursos gerados e captados: os recursos gerados (obtidos por meio de serviços prestados às empresas ou outras organizações de C, T \& I com fins particulares) e os recursos captados (obtidos por meio de convênios junto a agências de fomento para fins específicos) têm uma natureza própria, com foco na execução do serviço ou projeto aprovado que, no futuro, gerará impactos sobre o orçamento do Contrato de Gestão.

Nota-se que a regularidade dos orçamentos operacionais é identificada por todos os entrevistados como um fator crítico para o desempenho do LNLS.

A categoria CAT 07 - Eficiência gerencial apresentou quatro unidades de significado: i) modelo gerencial; ii) cenário de incertezas (capacidade de adaptação); iii) atuação na geração do conhecimento; iv) difusão científica e tecnológica.

Notadamente, as medidas de identificação da eficiência gerencial são relativamente complexas, mas entendidas pelos entrevistados como essenciais para atingir níveis superiores de resultados. O entrevistado LG 1 ressalta que "Isso é importante [a eficiência gerencial], muito mais a organização do que a instabilidade [orçamentária]". 
Modelo gerencial: é apontado como um ponto importante, e a questão da burocratização mínima é destacada, assim como a necessidade de modelos de governança que permitam uma operação descentralizada.

Cenário de incertezas: a incerteza é inerente à atividade de pesquisa, desenvolvimento e inovação, no entanto, os entrevistados destacaram que o modelo gerencial deve permitir a criação de mecanismos de estabilidade financeira visando a continuidade das atividades institucionais, seja em sua área de atuação, seja para aproveitar novas oportunidades identificadas.

Atuação na geração do conhecimento: outro aspecto mencionado pelos entrevistados relaciona-se à flexibilidade para a gestão do conhecimento gerado pela organização de modo a propiciar a apropriação institucional desse aprendizado.

Difusão científica e tecnológica: pode-se constatar uma preocupação com difusão científica e tecnológica como forma de ampliação da legitimidade institucional e de busca de novas oportunidades de interação nas áreas de atuação do LNLS.

A identificação das principais medidas de desempenho utilizadas pelo LNLS e a proposição de novos indicadores e elementos de acompanhamento que compõem as principais medidas de desempenho que devem ser monitoradas são apresentadas na sequência deste estudo.

\subsection{Análise dos indicadores de desempenho no período de 2006 a 2009}

Para identificar as principais medidas de desempenho que devem ser monitoradas pelo LNLS foram analisados, além do conteúdo das entrevistas, os indicadores formais de desempenho utilizados durante o período de 2006 a 2009. As informações estão contidas no Contrato de Gestão e respectivos Termos Aditivos, disponíveis no site <www.lnls.br $>$.

O quadro 2 apresenta os principais indicadores formais pactuados pelo LNLS.

Quadro 2

Indicadores do período de 2006 a 2009

\begin{tabular}{|lc|}
\hline \multicolumn{1}{|c|}{$\begin{array}{cc}\text { PRINCIPAIS INDICADORES } \\
\text { 2006-09 }\end{array}$} & FONTE \\
\hline Número de horas - linha & Ind. 1 \\
Número de pesquisadores externos treinados & Ind. 2 \\
Confiabilidade (horas entregues/horas previstas) & Ind. 3 \\
Desempenho da Fonte de Luz Síncrotron & Ind. 4 \\
Horas de estudo de máquinas e comissionamento & Ind. 5 \\
Grau de saturação no uso da Fonte de Luz Síncrotron & Ind. 6 \\
Custo por proposta realizada nas instalações abertas & Ind. 7 \\
Número de propostas realizadas & Ind. 8 \\
\hline
\end{tabular}




\begin{tabular}{|c|c|}
\hline $\begin{array}{l}\text { PRINCIPAIS INDICADORES } \\
2006-09\end{array}$ & FONTE \\
\hline Índice de ocupação das linhas de luz & Ind. 9 \\
\hline Índice de satisfação dos usuários & Ind. 11 \\
\hline Número total de publicações & Ind. 12 \\
\hline Publicações em revistas com fator de impacto maior do que 5 & Ind. 13 \\
\hline Publicações resultantes por pesquisador da ABTLuS & Ind. 14 \\
\hline Taxa de orientação de pós-graduados & Ind. 15 \\
\hline Taxa de supervisão de pós-doutores & Ind. 16 \\
\hline Número de memorandos técnicos disponíveis na Internet & Ind. 17 \\
\hline Número de projetos de desenvolvimento tecnológico & Ind. 18 \\
\hline Número de técnicos externos treinados & Ind. 19 \\
\hline Horas de treinamento por funcionário & Ind. 21 \\
\hline Alavancagem de recursos do Contrato de Gestão & Ind. 22 \\
\hline Horas de treinamento de técnicos externos treinados & Ind. 19.1 \\
\hline Horas de treinamento de pesquisadores externos treinados & Ind. 20.1 \\
\hline Número de horas destinadas aos usuários de luz síncrotron & Relatório \\
\hline Evolução do orçamento do Contrato de Gestão & Relatório \\
\hline Relação de propostas por área científica & Relatório \\
\hline Propostas realizadas por região do Brasil e outros países & Relatório \\
\hline Recursos provenientes da atividade de interação com o setor produtivo & Relatório \\
\hline Horas de microscópios eletrônicos & Relatório \\
\hline
\end{tabular}

Fonte: Relatórios Anuais e Contrato de Gestão. Elaborado pelos autores.

A partir da matriz de análise dos indicadores do período de 2006 a 2009 e das entrevistas de campo foi elaborada uma proposição de um conjunto de indicadores para o acompanhamento do desempenho do LNLS apresentados no quadro 3 e discutidos nas próximas seções.

\subsection{Proposição de indicadores e elementos de acompanhamento estratégico}

Para a formulação do modelo, foram identificadas duas perspectivas para as medidas de acompanhamento: i) a estratégica propriamente dita e ii) a de indutores de resultados.

As medidas de acompanhamento da estratégia foram relacionadas com as categorias:

\section{Legitimidade}

2. Resultados científicos

3. Confiabilidade das instalações

4. Disponibilidade das instalações 
As medidas de acompanhamento dos indutores de resultados foram compostas pelas seguintes categorias:

\section{Recursos humanos}

\section{Recursos financeiros}

\section{Eficiência gerencial}

As medidas para o acompanhamento da estratégia propriamente dita estão predominantemente caracterizadas no modelo atual; no entanto, novas medidas formais devem ser incorporadas para ampliação da legitimidade da organização, apesar da complexidade de mensuração. Nesse sentido, o presente trabalho pretende apontar algumas sugestões nessa direção, as quais não devem ser interpretadas como exaustivas.

Por outro lado, as medidas caracterizadas como indutoras de resultado, ou seja, aquelas que permitirão manter um ambiente favorável à geração de resultados no longo prazo não foram identificadas de forma efetiva no modelo atual. Dessa forma, o presente trabalho pretende contribuir para a redução desses gaps identificados.

Por fim, o quadro 3 resume a proposta dos elementos e indicadores.

Quadro 3

Proposta dos indicadores de acompanhamento do desempenho

\begin{tabular}{|c|c|c|}
\hline Categorias e Unidades de Significado & Elementos e Indicadores & Fonte \\
\hline \multicolumn{3}{|c|}{ Estratégia } \\
\hline \multicolumn{3}{|l|}{ Legitimidade } \\
\hline v Foco da missão institucional & $\begin{array}{l}\text { 1. Nível de incerteza dos cenários externos (relevância } \\
\text { das novas tecnologias) }\end{array}$ & Entrevista \\
\hline $\boldsymbol{\nabla}$ Monitoramento do ambiente de C\&T & 2. Cooperações internacionais & Entrevista \\
\hline v Indução de novas áreas de atuação & $\begin{array}{l}\text { 3. Novas tecnologias desenvolvidas (spin off de } \\
\text { tecnologias) }\end{array}$ & Entrevista \\
\hline v Competitividade e cooperação internacional & 4. Novas áreas de atuação induzidas & Proposição \\
\hline V Fronteira do conhecimento científico (ousadia) & 5. Percepção da comunidade científica e da sociedade & Entrevista \\
\hline $\begin{array}{l}\text { V Independência e integração das avaliações } \\
\text { externas }\end{array}$ & $\begin{array}{l}\text { 6. Capacidade de acompanhar as evoluções científicas } \\
\text { e tecnológicas }\end{array}$ & Entrevista \\
\hline \multicolumn{3}{|l|}{ Resultados Científicos } \\
\hline 1. Gerado por usuários externos & 1. Qualidade das publicações & Atual \\
\hline \multirow[t]{3}{*}{ 2. Pesquisa própria } & $\begin{array}{l}\text { 2. Publicações geradas pela existência da Fonte de } \\
\text { Luz Síncrotron }\end{array}$ & Entrevista \\
\hline & $\begin{array}{l}\text { 3. Posição relativa da pesquisa do LNLS no contexto } \\
\text { internacional }\end{array}$ & Entrevista \\
\hline & 4. Taxa de publicação por pesquisador interno & Atual \\
\hline
\end{tabular}




\begin{tabular}{|c|c|c|}
\hline Categorias e Unidades de Significado & Elementos e Indicadores & Fonte \\
\hline \multicolumn{3}{|c|}{ Estratégia } \\
\hline \multicolumn{3}{|l|}{ Confiabilidade das Instalações } \\
\hline 1. Principal (Fonte de Luz Síncrotron) & $\begin{array}{l}\text { 1. Disponibilidade de uso no tempo programado } \\
\text { (anel e estações experimentais) }\end{array}$ & Atual \\
\hline \multirow[t]{2}{*}{ 2. Instalações correlatas } & 2. Nível de satisfação dos usuários & Atual \\
\hline & 3. Capacidade de instrumentação científica & Entrevista \\
\hline \multicolumn{3}{|l|}{ Disponibilidade das Instalações } \\
\hline 1. Laboratório Nacional & 1. Inovações introduzidas por demanda dos usuários & Proposição \\
\hline 2. Laboratório Aberto & $\begin{array}{l}\text { 2. Uso das instalações por usuários externos (por } \\
\text { regiões do país e exterior) }\end{array}$ & Atual \\
\hline \multirow[t]{5}{*}{ 3. Laboratório Multiusuário e multidisciplinar } & 3. Taxa de renovação dos grupos de usuários & Entrevista \\
\hline & $\begin{array}{l}\text { 4. Experimentos realizados em multi-instalações e } \\
\text { multidisciplinares }\end{array}$ & Entrevista \\
\hline & 5. Projetos de pesquisa realizados & Proposição \\
\hline & $\begin{array}{l}\text { 6. Taxa de continuidade dos grupos de pesquisas } \\
\text { externos }\end{array}$ & Proposição \\
\hline & 7. Nível de efetividade da pesquisa realizada & Proposição \\
\hline \multicolumn{3}{|c|}{ Indutores de Resultados } \\
\hline \multicolumn{3}{|l|}{ Recursos Humanos } \\
\hline 1. Atração & $\begin{array}{l}\text { 1. Atratividade de recursos humanos em áreas } \\
\text { estratégicas }\end{array}$ & Entrevista \\
\hline 2. Retenção & $\begin{array}{l}\text { 2. Retenção de recursos humanos em áreas } \\
\text { estratégicas }\end{array}$ & Entrevista \\
\hline \multirow[t]{3}{*}{ 3. Capacitação } & 3. Rotatividade de recursos humanos qualificados & Proposição \\
\hline & 4. Nível de satisfação do pessoal interno & Entrevista \\
\hline & 5. Treinamento de usuários e de funcionários & Atual \\
\hline \multicolumn{3}{|l|}{ Recursos Financeiros } \\
\hline 1. Recursos do Contrato de Gestão & 1. Estabilidade orçamentária & Entrevista \\
\hline \multirow{5}{*}{$\begin{array}{l}\text { 2. Recursos gerados e captados extra Contrato de } \\
\text { Gestão }\end{array}$} & 2. Continuidade do fluxo de recursos & Entrevista \\
\hline & 3. Nível de investimento & Entrevista \\
\hline & 4. Custo de operação das instalações abertas & Atual \\
\hline & $\begin{array}{l}\text { 5. Alavancagem de recursos com projetos de fontes } \\
\text { diversas }\end{array}$ & Atual \\
\hline & 6. Novos projetos aprovados em agências de fomento & Proposição \\
\hline \multicolumn{3}{|l|}{ Eficiência Gerencial } \\
\hline 1. Modelo gerencial & 1. Qualidade e flexibilidade da gestão & Entrevista \\
\hline $\begin{array}{l}\text { 2. Cenário de incertezas (capacidade de } \\
\text { adaptação) }\end{array}$ & 2. Projetos realizados no prazo e orçamento pactuado & Entrevista \\
\hline 3. Atuação na geração do conhecimento & 3. Inovações introduzidas nas instalações abertas & Entrevista \\
\hline \multirow[t]{2}{*}{ 4. Difusão científica e tecnológica } & 4. Equilíbrio do portfólio de projetos de investimento & Proposição \\
\hline & 5. Inserção da comunicação nos grupos de interesse & Proposição \\
\hline
\end{tabular}

Fonte: Elaborado pelos autores. 
As medidas atuais de acompanhamento formal da entidade não apresentam aderência com a categoria denominada legitimidade. Dessa forma, essa categoria é composta por seis elementos ou indicadores de monitoramento que emergiram das entrevistas, sendo um deles por sugestão dos pesquisadores.

Os elementos nível de incerteza dos cenários externos e capacidade de acompanhar as evoluções científicas e tecnológicas devem ser acompanhados pela atuação dos grupos de pesquisa da entidade e, principalmente, pela atuação do corpo diretivo com forte influência das lideranças científicas.

Por outro lado, as cooperações internacionais, as novas tecnologias desenvolvidas e as novas áreas de atuação induzidas podem ser acompanhadas de forma quantitativa. Deve-se destacar a importância de medidas qualitativas das cooperações e das tecnologias desenvolvidas.

Por fim, o indicador da percepção da comunidade científica e da sociedade em geral deve ser formulado considerando as avaliações externas independentes e com uma pesquisa formal junto aos respectivos stakeholders, como usuários, governo e sociedade civil organizada.

A categoria resultados científicos apresentou maior aderência ao atual quadro de indicadores da entidade e relaciona-se mais intensamente com os mecanismos formais de acompanhamento da estratégia.

Em geral, os entrevistados foram enfáticos com relação à mensuração qualitativa dos resultados e à necessidade de ter uma pesquisa interna atuante e de fronteira, além da pesquisa gerada por usuários externos.

A categoria confiabilidade das instalações está presente no atual quadro de indicadores e relaciona-se com a atividade de laboratório aberto da instituição. No entanto, a forma de mensuração deve ser aprimorada para os indicadores de disponibilidade de uso e nível de satisfação dos usuários para maior utilidade no processo de tomada de decisão.

$\mathrm{O}$ indicador de capacidade de instrumentação científica foi apresentado como um mecanismo de diferenciação da instituição no seu papel de Laboratório Nacional e Aberto e sua mensuração é complexa.

As unidades de significado que compõe a categoria disponibilidade das instalações estão diretamente relacionadas com a missão base do LNLS. Os indicadores propostos complementam o atual quadro pactuado e propõem a inclusão de instalações correlatas, identificada pelas entrevistas como deficiência das atuais medidas.

A categoria recursos humanos prevaleceu com principal indutor de resultados e, apesar de apresentar dificuldades de mensuração, sugere-se que a instituição e o órgão supervisor discutam alternativas para um acompanhamento mais efetivo.

Os indicadores relacionados com a categoria recursos financeiros estão caracterizados de duas perspectivas: i) os recursos do Contrato de Gestão, principal fonte de recurso estável da entidade; ii) recursos extra Contato de Gestão, originados de projetos captados junto a agências de fomento ou gerados pela interação com outros atores de financiamento da pesquisa e desenvolvimento.

Para os recursos do Contrato de Gestão, os entrevistados destacaram a importância de estabilidade no fluxo de recursos, além da necessidade de elevação do patamar de financiamento público. 
Com relação aos recursos extra Contrato de Gestão, houve uma menor ênfase por parte dos entrevistados. No entanto, devem-se observar os níveis de alavancagem da instituição e sua capacidade de captação e geração de recursos no sistema de C, T \& I, com ênfase no papel dos Laboratórios Nacionais para a política de inovação sinalizada por sucessivos planos de governos.

A categoria eficiência gerencial deve se desdobrar em medidas internas de monitoramento. No entanto, sugere-se que as evidências dessa eficiência sejam explicitadas visando o seu acompanhamento.

Nesse sentido, os indicadores propostos devem representar um conjunto balanceado de medidas de acompanhamento, ou mesmo a qualificação da gestão por certificação externa. Importante destacar que não há atualmente, de forma explícita, medidas intermediárias desse monitoramento. Algumas medidas de monitoramento, como projetos realizados no prazo e orçamento pactuado e equilíbrio do portfólio de projetos de investimento, podem ser rapidamente adotadas, enquanto outras são mais complexas e exigirão recursos para a efetiva adoção.

Com base nessas evidências é sugerido um modelo de acompanhamento do desempenho a ser adotado pelo LNLS, conforme a figura 2.

Figura 2

Modelo de acompanhamento do desempenho

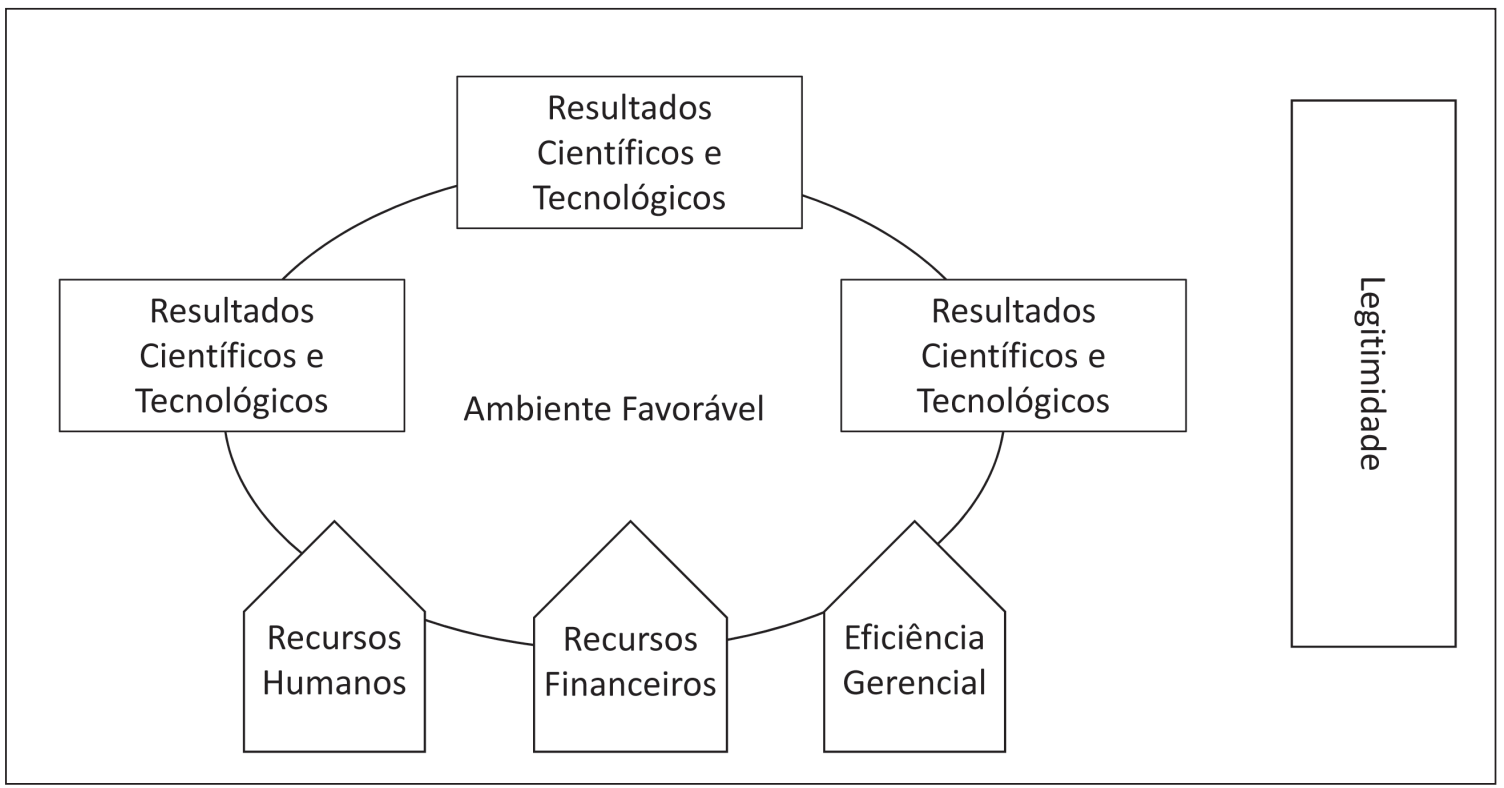

Fonte: Elaborada pelos autores.

O modelo conceitual para o acompanhamento das medidas de desempenho proposto considera que a geração de resultados científicos ou tecnológicos superiores, em organizações de C, T \& I, é fortemente influenciada por um ambiente favorável à pesquisa e desenvolvimento. 
Os principais fatores, identificados no presente trabalho, que são capazes de induzir ou favorecer a manutenção desse ambiente, estão relacionados com a capacidade da organização na gestão de recursos humanos e financeiros. Práticas de gestão mais eficientes também devem contribuir para a percepção de um ambiente diferenciado e propício ao desenvolvimento das organizações de $\mathrm{C}, \mathrm{T} \& \mathrm{I}$.

Entende-se que, para a atuação de Laboratórios Nacionais, outros dois elementos devem ser considerados: i) a credibilidade/confiabilidade das instalações e ii) o uso de forma aberta à comunidade científica, que permita a integração dos grupos de pesquisa da organização e dos pesquisadores-usuários externos, de forma a maximizar os resultados científicos ou tecnológicos.

No mais, sensores que caracterizem a legitimidade das missões das organizações de C, $\mathrm{T} \& \mathrm{I}$, devem ser incorporados às medidas de acompanhamento como medidas de impacto científico, tecnológico, social e econômico.

\section{Considerações finais}

O presente estudo teve como objetivo identificar as principais medidas de desempenho que devem ser monitoradas por organizações de C, T \& I, a partir de um estudo de caso realizado no LNLS, fundamentado em entrevistas de profundidade e interpretadas por meio de análise de conteúdo e com triangulação por meio de análise de documentos e observação.

As principais medidas de desempenho identificadas para o acompanhamento do desempenho de organizações de C, T \& I foram agrupadas em duas perspectivas: i) a da estratégia propriamente dita e ii) a de indutores de resultados.

As categorias identificadas nas medidas de acompanhamento da estratégia foram: i) legitimidade; ii) resultados científicos; iii) confiabilidade das instalações; iv) disponibilidade das instalações.

A categoria legitimidade institucional congrega os aspectos mais amplos da organização, sendo necessário um monitoramento no nível estratégico da entidade. Nesse aspecto, as avaliações externas sistemáticas contribuem para o referencial de desempenho.

Os resultados científicos, em organizações de C, T \& I, são os monitorados por indicadores consolidados no meio acadêmico, mas ficou evidenciado que a mensuração qualitativa desses resultados não é trivial e sugere-se a introdução de novos indicadores para que o LNLS possa acompanhar esse parâmetro de desempenho.

Para a atuação dos Laboratórios Nacionais, com a característica do LNLS, foram identificadas as categorias confiabilidade e disponibilidade das instalações. As medidas de acompanhamento atual são bastante aderentes a essas categorias e os pontos de aprimoramento referem-se à ampliação da abrangência das medidas de confiabilidade das instalações e à formalização das medidas de uso de diversas instalações e multidisciplinares.

As medidas de acompanhamento denominadas indutores de resultados foram compostas pelas seguintes categorias: i) recursos humanos; ii) recursos financeiros; e iii) eficiência gerencial. 
A qualidade técnica da equipe de pesquisadores e técnicos foi identificada como um fator de atração e retenção de recursos humanos. Para alcançar nível de excelência, a formação de recursos humanos, tanto internos quanto externos, é imprescindível. Esse foi um ponto de atenção presente na maioria das entrevistas, e medidas mais efetivas de acompanhamento intermediário, por meio de indicadores de desempenho, são sugeridas no presente trabalho.

A estabilidade dos recursos financeiros foi considerada essencial para a geração de resultados de médio e longo prazo que singularizam as organizações de C\&T. A proposição de novos indicadores visa reduzir a assimetria de informações e explicitar as medidas indiretas de acompanhamento do desempenho financeiro. Nessa categoria, deve-se ampliar as discussões sobre as formas de financiamento e fomento para as atividades de Laboratórios Nacionais.

A percepção de perda de eficiência gerencial e da flexibilidade operacional também foi apontada como uma ameaça à legitimidade institucional e afeta o ambiente adequado para a geração de resultados em $\mathrm{C}, \mathrm{T} \& \mathrm{I}$.

A implementação de novos mecanismos de mensuração e de acompanhamento dos parâmetros propostos no presente trabalho provavelmente exigirá investimentos nos sistemas de controle gerencial da organização para permitir maior aderência dos indicadores pactuados com a estratégia organizacional.

Formulou-se um modelo conceitual para acompanhamento das medidas de desempenho para organizações de C, T \& I constituído de 36 indicadores, e destes apenas oito já constavam no Contrato de Gestão de 2006 a 2009.

Esse modelo requer ações para promover uma maior integração das atuais avaliações institucionais, identificadas principalmente pelo Comitê Científico e pela Comissão de Avaliação do Contrato de Gestão. Nesse aspecto, sugere-se, ainda, a adoção de um ciclo de avaliação estendido do próprio sistema de C, T \& I para legitimação da missão dessas organizações.

Os resultados desta pesquisa sugerem ainda que os parâmetros relacionados com as medidas quantitativas estão consolidados por meio do Contrato de Gestão firmado entre as partes. No entanto, nota-se a ausência de medidas qualitativas para o acompanhamento estratégico e sugere-se um painel de indicadores mais equilibrado e com capacidade de comparabilidade com outros laboratórios internacionais. Quanto aos aspectos qualitativos dos resultados científicos, estes, apesar de relativamente consolidados, precisam ser adaptados às características de um Laboratório Nacional.

A generalização dos resultados deve ser realizada com cautela na medida em que os fatos aqui relatados referem-se a um caso específico, entretanto podem ser úteis para outras instituições de ciência e tecnologia, em particular, àquelas cuja gestão esteja pautada no modelo de organização social.

Por fim, recomenda-se estudos que avaliem o sistema de ciência e tecnologia do Brasil com relação aos impactos sociais e tecnológicos introduzidos por essas organizações. 


\section{Referências}

ANTHONY, Robert N.; GOVINDARAJAN, Vijay. Sistemas de controle gerencial. São Paulo: Atlas, 2006.

BARDIN, Laurence. Análise de conteúdo. Lisboa: Edições 70 Ltda., 2004.

BIN, Adriana. Planejamento e gestão da pesquisa e da inovação: conceitos e instrumentos. Tese (doutorado) - Instituto de Geociências, Departamento de Política Científica e Tecnológica, Universidade de Campinas, Campinas, 2008.

BRASIL. Ministério da Ciência e Tecnologia. Academia Brasileira de Ciências. Livro verde. Brasília, 2001.

BURNS, Tom; STALKER, George M. The management of innovation. Londres: Tavistock, 1961.

CHIESA, Vittorio et al. How do measurement objectives influence the R\&D performance measurement system design?: evidence from a multiple case study. Management Research News, v. 30, n. 3, p. 187-202, 2007.

CONTRATO de gestão 2011-16. Disponível em: <www.cnpem.br/wp-content/documents/Contrato_de_Gestao_Set2010>. Acesso em: 20 fev. 2013.

DONALDSON, Lex. Teoria da contingência estrutural. In: CLEGG, Stewart R.; HARDY, Cynthia; NORD, Walter R. (Org.). Handbook de estudos organizacionais. 1. ed. 4. reimp. São Paulo: Atlas, 2007. v. 1, p. 104-134.

ETHERINGTON, Lois; TJOSVOLD, Dean. Managing budget conflicts: contribution of goal interdependence and interaction. Revue Canadienne des Sciences de l'Administration, v. 15, n. 2, p. 142-151, jun. 1998.

FERREIRA, Aldonio; OTLEY David. The design and use of performance management systems: an extended framework for analysis. Management Accounting Research. v. 20, n. 4, p. 263-282, dez. 2009.

FRANCO-SANTOS, Monica; LUCIANETTI, Lorenzo; BOURNE, Mike. Contemporary performance measurement systems: a review of their consequences and a framework for research. Management Accounting Research, v. 23, n. 2, p. 79-119, jun. 2012.

GIBBONS, Michael et al. The new production of knowledge: the dynamics of science and research in contemporary societies. Londres: Sage Publications Inc., 1994.

KAPLAN, Robert S.; NORTON, David P. The balanced scorecard: translating strategy into action. Boston: Harvard Business School, 1996.

MALMI, Teemu; BROWN, David A. Management control systems as a package - opportunities, challenges and research directions. Management Accounting Research, v. 19, n. 4, p. 287-300, dez. 2008.

MALINA, Mary A.; SELTO, Frank H. Choice and change of measures in performance measurement models. Management Accounting Research, v. 4, n. 15 p. 441-469, dez. 2004. 
MERCHANT, Kenneth A.; VAN DER STEDE, Wim A. Management control systems: performance measurement, evaluation and incentives. Londres: Pearson; Prentice Hall, 2007.

NEELY, Andy et al. Strategy and performance: getting the measure of your business. 1. ed. Cambridge: Cambridge University Press, 2002.

NEUENDORF, Kimberly A. The content analysis guidebook. California, USA: Sage Publictions, 2002.

OYADOMARI, José C. T. Uso do sistema de controle gerencial e desempenho: um estudo em empresas brasileiras sob a ótica VBR (Visão Baseada em Recursos). Tese (doutorado) — Faculdade de Economia, Administração e Contabilidade, Universidade de São Paulo, São Paulo, 2008.

PEREZ, Gilberto. Adoção de inovações: um estudo de caso sobre o uso de sistema de informação na área de saúde. Tese (doutorado) - Faculdade de Economia, Administração e Contabilidade, Universidade de São Paulo, São Paulo, 2006.

PORTER, Michael E. Vantagem competitiva. Rio de Janeiro: Campus, 1989.

SCHWARTZMAN, Simon. A pesquisa científica e o interesse público. Revista Brasileira de Inovação, v. 1, n. 2, p. 361-395, jul./dez. 2002.

SIMONS, Robert. Levers of organization design. Boston: Harvard Business School Publishing, 2005.

Eduardo Frare é mestre em controladoria pela Universidade Presbiteriana Mackenzie, gerente de Planejamento e Avaliação do Centro Nacional de Pesquisa em Energia e Materiais e professor da PUC-Campinas. E-mail: eduardo.frare@cnpem.br.

Ricardo Lopes Cardoso é doutor em ciências contábeis pela Universidade de São Paulo, professor do Programa de Pós-Graduação em Ciências Contábeis da Universidade Presbiteriana Mackenzie. E-mail: ricardo.cardoso@mackenzie.br.

José Carlos Tiomatsu Oyadomari é doutor em ciências contábeis pela Universidade de São Paulo, professor do Programa de Pós-Graduação em Ciências Contábeis da Universidade Presbiteriana Mackenzie e professor do Insper Instituto de Ensino e Pesquisa. E-mail: oyadomari@mackenzie.br.

Octavio Ribeiro de Mendonça Neto é doutor em ciências contábeis pela Universidade de São Paulo, professor do Programa de Pós-Graduação em Ciências Contábeis da Universidade Presbiteriana Mackenzie. E-mail: octavio.mendonca@mackenzie.br. 